\title{
Type Ia Supernova Cosmology
}

\author{
B. Leibundgut - M. Sullivan
}

Received: date / Accepted: date

\begin{abstract}
The primary agent for Type Ia supernova cosmology is the uniformity of their appearance. We present the current status, achievements and uncertainties. The Hubble constant and the expansion history of the universe are key measurements provided by Type Ia supernovae. They were also instrumental in showing time dilation, which is an direct observational signature of an expansion. Connections to explosion physics are made in the context of potential improvements of the quality of Type Ia supernovae as distance indicators. The coming years will see large efforts to use Type Ia supernovae to characterise dark energy.
\end{abstract}

\section{Background}

The monolithic picture of Type Ia supernovae (hereafter SNe Ia) of a decade ago has undergone significant refinements. The observational diversity of SNe Ia includes ranges in luminosity, light-curve shape, colour, spectral evolution, and host galaxy environment (e.g. see Branch \& Wheeler 2017, Maguire 2016 and Taubenberger 2016 for summaries on SNe Ia). Although almost certainly thermonuclear explosions of carbon-oxygen white dwarf stars, in detail we now believe that SNe Ia are the result of possibly different explosion mechanisms after a variety of evolutionary paths towards the explosion. This has opened a rich research field to understand binary stellar evolution and explosion physics. At the same time, the observed diversity

B. Leibundgut

European Southern Observatory, Karl-Schwarzschild-Strasse 2, D-85748 Garching, Germany and Excellence Cluster Universe, Technische Universität München, Boltzmannstrasse 2, D-85748 Garching, Germany

E-mail: Bruno.Leibundgut@eso.org

M. Sullivan

Department of Physics and Astronomy, University of Southampton, Southampton, Hampshire, SO17 1BJ, UK

E-mail: M.Sullivan@soton.ac.uk 
has occasionally raised doubts as to whether the cosmological results of SNe Ia are reliable.

SNe Ia are used in three specific areas of cosmology. Historically, they have been employed as an accurate distance indicator to determine the local expansion rate of the Universe (e.g. Leibundgut 2016, Saha and Macri 2016, and references therein), to demonstrate that cosmological redshifts and time dilation are connected as predicted in all theories based on general relativity (Leibundgut et al. 1996, Riess et al. 1997, Goldhaber et al. 2001, Blondin et al. 2008), and to map the expansion history of the universe, which led to the original discovery of the accelerated expansion (Riess et al. 1998, Perlmutter et al. 1999), and more recent studies investigating the nature of dark energy (Betoule et al. 2014, for reviews see Leibundgut 2001, Perlmutter \& Schmidt 2003, Leibundgut 2008, Goobar \& Leibundgut 2011). The distance determination is critical for the Hubble constant and the expansion history, while the uniformity of the light curves and spectral evolution was used to show how time dilation acts on distant supernovae.

Establishing the uniformity of SNe Ia has been an important factor in their cosmological application. SNe Ia are observed to explode with approximately the same luminosity to within around a factor of two, presumably due to the similarity of the triggering white dwarf mass and therefore the amount of nuclear fuel available to burn. More detailed observations have led to sophisticated treatments and standardisations, involving light curves, spectra, and the star-formation rate and stellar masses of the SN Ia host galaxies. The light-curve shape vs. luminosity relation as originally introduced by Phillips (1993) was the most important improvement to achieve distance accuracies required to detect the acceleration and to bring the uncertainty in the determination of $\mathrm{H}_{0}$ to less than $5 \%$ (see Phillips and Burns 2016, for a review on the different light curve correction methods). Today, the most popular correction methods are SALT2 (Guy et al. 2007), MLCS2k2 (Jha et al. 2007), SNooPy (Burns et al. 2011) and SiFTO (Conley et al. 2008). There are differences in how these methods apply the corrections and in their training sets, but the results appear quite robust and only subtle differences remain.

One critical parameter is how reddening is implemented: this is a free parameter combining colour and absorption in the cosmological interpretation of SALT2 and SiFTO (e.g. Tripp 1998), but follows an astrophysical reddening law in MLCS2k2 (albeit with a non-standard $R_{V}$ ) and SNooPy. While these simple approaches are adequate for the current generation of experiments, they are unlikely to be sufficient as SN Ia measurements become ever more precise. Recent studies have provided evidence that the colour-luminosity parameter (' $\beta$ ') may evolve with redshift (e.g. Shariff et al. 2016); evidence that the SN Ia colour corrections can produce biased parameters if the underlying SN colour distribution is not accurately known (e.g. Scolnic \& Kessler 2016); evidence for diversity in the extinction laws affecting SNe Ia (Amanullah et al. 2015); and long-standing evidence that conflating SN extinction and intrinsic colour variation into one parameter can (as might be expected) produce biased results(e.g. Mandel et al. 2017). 
These effects will lead to the handling of SN colour becoming a dominant uncertainty in upcoming analyses.

The most direct and easiest way to show that SNe Ia yield good (relative) distances is the Hubble diagram. Good standard candles delineate a line with a fixed slope, given by the linear expansion in the local universe $(z<0.1)$, when plotting observed magnitude versus the logarithm of the redshift $(m \propto 5 \log (z)$; see Fig. 1). The zeropoint of the expansion line is a combination of the Hubble constant and the absolute magnitude of the distance indicator. The scatter around this slope gives an indication on the precision of the standard candle. After correction by the methods mentioned above, SNe Ia provide individual distances of better than $6 \%$ (e.g. Riess et al. 2016, Dhawan et al. 2018). This argument is completely independent of any knowledge of the physical nature of SNe Ia and can be regarded as entirely empirical. The Hubble diagram has traditionally been used to establish the quality of distance indicators (see the reviews by Branch 1982, 1998, Branch \& Tammann 1992).

Although nearly all published cosmological constraints have been made with distance estimators constructed in the rest-frame optical, redder wavelengths have the potential to provide superior distance estimates. Fig. 1 shows the rest-frame $J$-band Hubble diagram of nearby $\mathrm{SNe}$ Ia (from Dhawan et al. 2018; a discussion of earlier SNe Ia infrared Hubble diagrams can be found in Krisciunas 2016). In this case no correction to the $J$-band peak magnitude has been applied: the SNe Ia have been treated as pure standard candles. The slope of the line has been fixed to the fiducial slope for the linear expansion. The fit is extremely good with a scatter around the Hubble line of 0.12 magnitudes. It is clear from the uncertainties for each supernova that the scatter is intrinsic to the SN Ia sample and a suitable correction might decrease it further. However, there is no clear correlation with other observables (colour, light-curve shape in the optical bands, host galaxy properties; see Dhawan et al. 2018 for a discussion). The scatter should be compared to a standard variation of 0.128 magnitudes for 271 optical light curves with $0.01<z<0.15$ used in Riess et al. (2016).

A second method to establish the uniformity of a (normalised) luminosity is by sampling SNe Ia at known distances. If the used distances are absolutely calibrated, then this method is more powerful than the Hubble diagram. Measuring exact distances to $\mathrm{SNe}$ Ia has the problem that the volume reachable by reliable distance indicators is rather limited and the low rate of SNe Ia leads to very small samples. The Hubble Space Telescope (HST) was critical for this method (see Saha and Macri 2016, for a review of these programs) and has by now yielded a sample of $19 \mathrm{SNe}$ Ia with Cepheid distances (Riess et al. 2016). The Cepheids were measured in the near-infrared $(1.6 \mu \mathrm{m})$ to reduce uncertainties in phase calibrations and absorption. The standard deviation among the absolute luminosities of these 19 SNe Ia with Cepheid distances is 0.17 magnitudes. This is larger than the scatter in the relative distances measured through the Hubble diagram (as already pointed out by Dhawan et al. 2018). Nevertheless, this means that the SNe Ia in the calibrator set show individual distance uncertainties of about $8 \%$. 


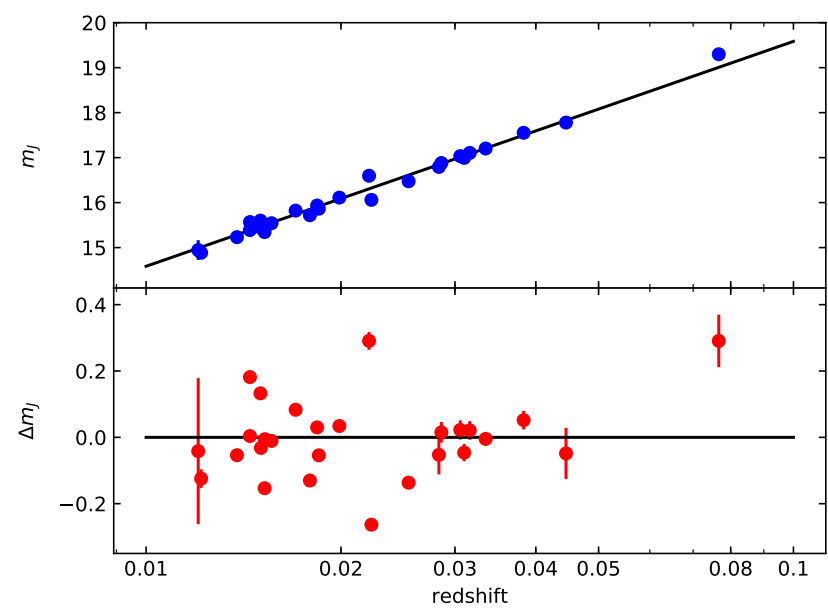

Fig. 1 The Hubble diagram of SNe Ia in the rest-frame $J$-filter. No correction has been applied to the maximum magnitudes. The slope of the line is fixed by the linear expansion in the local universe. Data from Dhawan et al. (2018).

These two independent methods show that SNe Ia currently provide the most accurate individual distances at cosmological scales. Even though the physics of explosion and radiation transport may not be fully understood, the observed uniformity of the light curves and the empirical normalisation methods yield an excellent consistency in the local universe.

\section{SN Ia cosmology}

As already indicated, SNe Ia play decisive roles for the determination of the Hubble constant and the mapping of the expansion history of the universe and hence the contribution of various energy components to the cosmic expansion.

\subsection{Hubble constant}

SNe Ia are the secondary distance indicators to step from the gravitationally bound systems, like the Local Group and the Virgo Supercluster, into the Hubble flow, where the cosmic expansion dominates. Once the best-fit expansion line in the Hubble diagram is determined, the Hubble constant can be found from the line zeropoint and an independent measurement of the absolute magnitude of SNe Ia at maximum $\left(M_{\max }\right)$. Attempts to derive the absolute magnitude from models have so far failed due to insufficient knowledge of the explosions and the variety displayed amongst SNe Ia (e.g. Stritzinger \& Leibundgut 2005). 
The direct way of measuring $M_{\max }$ is through Cepheid distances, which themselves are anchored by geometric methods. This has been the classic distance ladder approach (see Jacobi et al. 1992 for a comprehensive description of early methods). The HST key program (Freedman et al. 2001) and the SN Ia programs by Sandage, Tammann and Saha, and $\mathrm{SH}_{0} \mathrm{ES}$ (see Saha and Macri 2016, for a complete bibliography of these efforts) have reduced the distance ladder to three steps: a local (geometric) anchor to calibrate the Cepheid period-luminosity (Leavitt) relation, calibrate SNe Ia with Cepheid distances and then use the Hubble diagram to step into the Hubble flow. Most prominent anchors are geometric distances to Milky Way Cepheids, eclipsing binaries in the Large Magellanic Clouds and the water megamasers in NGC 4258 (Humphreys et al. 2013). By reducing the number of steps in the distance ladder the sources of uncertainty have been diminished significantly. Nevertheless, there remain several systematic uncertainties, which will need to be addressed in the future. Among them are the influence of metallicity on the Leavitt relation of the Cepheids and some slight incompatibilities of the different anchors. It is a great achievement that the Hubble constant has been determined to a few percent!

\subsection{Time dilation}

Universe models based on General Relativity predict time dilation due to the expansion of the universe. Non-expanding models typically do not predict time dilation for distant objects. The SN Ia light evolution can be used as accurate 'clock' to measure time dilation in distant objects. This had been suggested already shortly after supernovae were discovered as a class (Wilson 1939) and was confirmed only some 50 years later, first through light curves (Leibundgut et al. 1996, Goldhaber et al. 2001) and then in the spectral evolution (Riess et al. 1997, Blondin et al. 2008). Fig. 2 shows a comparison of the observed $B$ light curves of nearby SNe Ia $(z<0.1)$ and observed $R$ light curves of SNe Ia from the JLA sample with $0.45<z<0.55$. The $B$ filter is redshifted into $R$ at $z \sim 0.5$ (e.g. Schmidt et al. 1998). Despite considerable diversity in light curve shape that can be seen in the local and the distant SN Ia samples, the high-redshift SNe Ia clearly have a wider peak and a significantly larger rise time. These are the signatures of time dilation. Quantitative analyses confirm the expected factor of $1+z$ (Leibundgut et al. 1996, Goldhaber et al. 2001, Blondin et al. 2008). All distant supernova light curves are now routinely corrected for time dilation.

\subsection{Cosmic expansion history}

SNe Ia are the most mature and well-exploited probe of cosmic acceleration, and remain a highly-competitive and immediate route to constraining its nature. In a homogeneous and isotropic universe, i.e. using the RobertsonWalker metric, the scale factor changes according to the energy content of the universe. In older formulations, the deceleration parameter was determining the fate of the universe (e.g. Sandage 1961) and cosmology was 


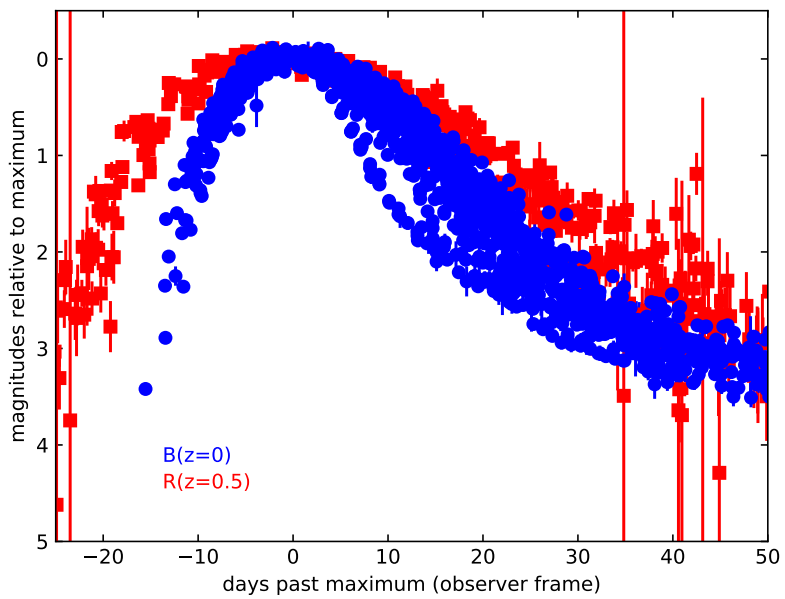

Fig. 2 Observed light curves of SNe Ia in the local universe $(z<0.1)$ in $B$ and at a redshift $0.45<z<0.55$ in $R$. The normalisation is at the time of maximum and the individual light curves have been shifted to the same maximum brightness. The stretching of the observed $R$ light curves relative to the local $B$ light curve is apparent.

referred to as the search for two numbers $\left(H_{0}\right.$ and $q_{0}$; Sandage 1970). The discovery of the accelerated expansion (Riess et al. 1998, Perlmutter et al. 1999) and the corresponding existence of Dark Energy (in FriedmannLemaitre models) has been widely documented. The simple argument on the accuracy of the distance indicator used in the local Hubble diagram cannot be applied at large distances, since deviations from the linear expansion are the measurement. Critical assumptions for this application are that the corrections applied to the nearby SNe Ia are valid throughout the observed redshift range, that no selection biases affect the distant samples and that the corrections for redshift and absorption can be controlled. A thorough discussion of many of these fundamental assumptions can be found in Goobar \& Leibundgut (2011).

When combined with Planck CMB measurements and Baryon Acoustic Oscillation results, current SN Ia samples measure the average dark energy equation-of-state parameter $w$ to around $4-4.5 \%$. The state of the art until recently was the combination of the Sloan Digital Sky Survey-II (SDSS-II) SN program and the first three years of the five-year CanadaFrance-Hawaii Telescope Supernova Legacy Survey (SNLS), plus various other sources (Betoule et al. 2014). They presented a sample of 740 spectroscopically confirmed SNe Ia from $0.1<z<1$ (374 from SDSS, 242 from SNLS). The joint light-curve analysis (JLA) in Betoule et al. (2014) has become the default for cosmological applications of $\mathrm{SNe} \mathrm{Ia}$, is most frequently used in comparison with other methods to measure the cosmological parameters (e.g., Planck; Planck Collaboration et al. 2016) and is 
the de facto standard of SN Ia cosmology. The cosmological analysis of the full five-year SNLS and the ESSENCE SN Ia search (Narayan et al. 2016, 213 spectroscopically confirmed SNe Ia) have not yet been presented. Since the systematics of the photometric systems are critical, it is very difficult to combine the SNLS and the ESSENCE data sets for a cosmological analysis. So far, no attempt has been made to combine these data sets. Underlying this problem is the difficulty of absolute calibration of the photometric systems between the different telescopes and instruments (e.g. Scolnic et al. 2014) and the subsequent uncertainties in colour, reddening and $\mathrm{K}$-corrections.

New attempts to combine large SN Ia samples have been made by Scolnic et al. (2017) and Jones et al. (2018). By adding 276 spectroscopically confirmed SNe Ia from the Pan-STARRS1 Medium Deep Survey to the JLA sample, the total number of SNe Ia in the analysis increases to 1049 objects. The conclusions remain unchanged and the uncertainties have decreased slightly. For a constant equation of state parameter $\omega$ in a flat universe the uncertainty is now about $4 \%$.

With these large samples, it has been clear for several years that the results are now dominated by experimental systematic uncertainties (Conley et al. 2011), and reducing the statistical error with a larger sample of the same type will only provide limited improvement over the current result. This also means that new data samples based on the same experimental methodology, in particular photometric calibration of the observed optical SN Ia fluxes, will not yield improvements in accuracy.

The SN Ia likelihoods are typically orthogonal to other measurements of cosmological parameters (e.g. Bahcall et al. 1999) because of the lower mean redshift compared to most other methods (see Goobar \& Perlmutter 1995, for an illustration of this effect). SNe Ia currently also sample the redshift space out to $z \approx 1.4$ more densely than any other method. While the CMB provides a snapshot at $z=1000$, Baryonic Acoustic Oscillation, weak lensing and redshift space distortions typically provide measurements at a few redshifts. The latter methods rely on large statistical samples to overcome the considerable individual distance uncertainties. The accuracy to a few percent in distances have allowed the SNe Ia to provide a much denser sampling with a significantly smaller investment of observing time.

\section{SN Ia physics}

Taken at face value, the simplicity of the SN Ia technique in cosmological applications - comparing the relative brightnesses of events at different redshifts - suggests the ultimate accuracy of their use may only be limited by the extent to which relative calibrations of their fluxes can be performed. But this is an oversimplification, and ignores the considerable ignorance of the detailed underlying physics governing SN Ia explosions.

The compactness of the progenitor stars of SNe Ia has been demonstrated by the non-detection in deep pre-explosion imaging of the nearest, unobscured SN Ia in decades, SN 2011fe (Nugent et al. 2011, Li et al. 2011). The progenitor star is very likely in a binary system, in which the 
progenitor white dwarf can be disturbed, or gain mass, and thus explode. But the configuration of this progenitor system prior to explosion remains uncertain.

Searches for compact objects in young SN remnants have so far come up empty handed, indicating that the secondary star is most likely destroyed (e.g. Kerzendorf et al. 2017). There are indications that some SNe Ia appear associated with circumstellar gas (Patat et al. 2007, Sternberg et al. 2011, Maguire et al. 2013, Sternberg et al. 2014, Cikota et al. 2017), which presumably came from a companion to the white dwarf. Sometimes this circumstellar material can be very dense, and interactions with it almost drown out the classical SN Ia signature (e.g., Dilday et al. 2012). But the majority of SNe Ia are consistent with originating from systems of (at least) two white dwarfs in clean environments, with no indication of circumstellar material (Phillips et al. 2013).

The main observational characteristics of SNe Ia are the light curve shapes, which reflect a combination of explosion energy, nickel mass, ejecta mass and composition, and spectral evolution, which traces the ejecta structure and element stratification, if present. The variety of SNe Ia has been described in Maguire (2016), Taubenberger (2016) and Jha (2017).

The majority of SN Ia display very similar characteristics. In particular, the success in applying the Phillips relation for light-curve shape leading to a small scatter in the Hubble diagram, is testimony to the uniformity of a majority of the explosions. There appears to be a continuity in the distribution of light-curve widths and peak luminosity. This has been connected to a possible diversity in the nickel mass produced in the explosion (Nugent et al. 1995, Contardo et al. 2000, Dhawan et al. 2016). A possible connection to the ejecta mass was established later (Stritzinger et al. 2006, Scalzo et al. 2014a,b).

Several distinctions have been introduced among the fast-declining SNe Ia. Taubenberger (2016) provides an exhaustive overview. Many different behaviours have been observed and no clear consensus on a possible physical interpretation of the diversity has emerged.

Several special sub-classes of SNe Ia have been identified. One such are the SNe Iax (Foley et al. 2013, Jha 2017). They have originally been identified through the observations of the peculiar SN 2000cx (Li et al. 2001) and SN 2002cx (Li et al. 2003). SNe Iax are SNe Ia with a faster evolution (fast rise-times and typically also a fast decline-rate after maximum) and low expansion velocities (see Jha 2017, for an overview of SN Iax properties). Most importantly, these SNe Ia show a lower luminosity when compared to most other SNe Ia. Another distinguishing characteristic is the lack of a second maximum in the near infrared light curves. It has been suggested that SNe Iax come from pure deflagration explosions (e.g. Phillips et al. 2007) and it seems that at the low luminosity end of the SN Ia distribution a different explosion mechanism might be in place than for the regular-luminosity objects (Dhawan et al. 2017). Some SNe Iax might not completely incinerate and leave a stripped white dwarf behind (Fink et al. 2014). SNe Iax can be cleanly separated from regular SNe Ia and are eliminated from SN cos- 
mology samples. Due to their lower luminosities they are also discriminated against in distant $\mathrm{SN}$ samples.

More problematic for cosmology are 'overluminous' SNe Ia. The first such object identified was SN 1991T (Filippenko et al. 1992, Phillips et al. 1992). While somewhat more luminous than the majority of SNe Ia, it is still considered to be connected to the bulk of SNe Ia. Its light-curve shape fits onto the Phillips relation, and its luminosity can be normalised. A separate class of 'super-Chandrasekhar' mass SNe Ia has been observed. The first example of these objects was SN 2003fg (Howell et al. 2006). The best observed super-Chandrasekhar SNe Ia appear to be SN 2007if and SN 2009dc (see Taubenberger 2016, for a summary of all known objects at the time). These overluminous SNe Ia are characterised by a long rise-time, which leads to estimates of nickel masses in excess of the Chandrasekhar mass (hence the name). They appear UV bright and display blue colours around maximum, but also are more luminous at infrared wavelengths, where most other SNe Ia reach rather similar peak luminosities. Super-Chandrasekhar mass SNe Ia are further characterised by lower ejecta velocities. Simple models predict ejecta masses up to 2 or $3 M_{\odot}$ (Taubenberger et al. 2011). It is not obvious how such superluminous $\mathrm{SNe}$ Ia can be identified in the cosmological samples. For a given redshift they would indicate a larger distance and hence could mimic acceleration, if they would represent a significant fraction of the most distant supernovae. Due to Malmquist bias one expects the fraction of luminous SNe Ia to increase with distance. However, the intrinsic rate of these events is believed to be very small - to date, no clear superluminous SNe Ia have been identified through their slow rise and the blue colours in the distant samples.

While understanding the SN Ia physics is exciting in its own right, it has no direct influence on the capability of (normal) SNe Ia to determine accurate distances. Knowing the exact explosion mechanism and the radiation transport involved in these explosions may provide additional refinements in the normalisation methods, like the light-curve shape and colour corrections and the potential dependencies on host galaxy properties. If different explosion mechanisms for subgroups of SNe Ia could be identified, then a cleaner sample might be constructed. However, the identification criteria need to be clear and simple so that they can be applied to noisy data from faint, distant objects.

\section{Sources of Uncertainty}

Cosmological results from SNe Ia depend on a number of systematic uncertainties. The statistical error has been reduced to rather small levels (about half the total uncertainty; Betoule et al. 2014). Reducing it further with increased samples will not overcome the systematic problems with the distance measurements of SNe Ia. An important limiting factor was the required accurate photometry of the SNe Ia. The latest projects have placed a strong focus on uniform photometry and have mostly used a single telescope/instrument combination to avoid cross-calibrations of different photometric systems. Major fundamental uncertainties are the normalisation 
of the luminosity through the light shapes, colours and host galaxy properties, which are coupled with the unknown absorption in the host galaxy and potential variations of the absorption law in different galaxies. The solution for colour, absorption and K-corrections are intimately connected and are difficult to disentangle.

From Section 3 it is also clear that a better understanding of the progenitor channels and the explosion physics will help in selecting clean SN Ia samples at all redshifts. Any secular evolution, e.g. potential dependencies of the peak luminosity on stellar metallicity, could influence the cosmological measurement. So far, they appear unlikely, but independent tests have been sparse. The main argument against a metallicity dependency is that the local SNe Ia cover a much wider range in metallicities than distant ones, where the average metallicity is presumably smaller. Hence the observed scatter would be largest for nearby SNe Ia. Also, the white dwarf produced in the stellar core by the nuclear burning should be mostly independent of metallicities.

A promising way forward are observations of SNe Ia in the rest frame near-infrared. At these wavelengths the absorption is greatly diminished and SNe Ia behave much more uniformly around maximum light (Krisciunas et al. 2004, Dhawan et al. 2015). The difficulty is to obtain restframe nearinfrared light curves of distant SNe as the light is redshifted into wavelength regions with high sky brightness essentially preventing detecing the SNe Ia beyond $z \approx 0.3$ from the ground. The best near-IR Hubble diagrams are from Freedman et al. (2009), Barone-Nugent et al. (2012), Stanishev et al. (2015) and Krisciunas (2016). Extending them to higher redshifts requires space observations. Euclid and in particular WFIRST will aim to obtain such measurements in the next decade and will significantly reduce the uncertainties due to absorption and colours.

\section{Current surveys}

Three distinct phases of SN Ia surveys can be discerned. The first, the early SN searches, were essentially trying to find as many $\mathrm{SNe}$ as possible, and establish them as good distance indicators (see Leibundgut 2016, for a historical account). During the 1980s and 1990s SNe Ia were mostly used to measure an accurate value of the Hubble constant (most notably the Calán/Tololo survey; Hamuy et al. 1996). Discovering distant SNe Ia and cosmic acceleration took most of the decade between 1988 and 1998 (see Garnavich 2017, for a summary), followed by another decade to confirm the discovery (Riess 2016, provides a historical overview).

The second phase has been concerned with establishing the machinery required to make the discovery of SNe Ia routine. Survey such as SNLS, SDSS-SN, and ESSENCE, together with large allocations of spectroscopic follow-up time on $8 \mathrm{~m}$-class facilities, made the discovery and confirmation of SNe Ia up to $z=1$ common place. This phase is responsible for the current state-of-the-art cosmological constraints.

The third phase, which we have just entered, is the establishment of samples of such size that spectroscopic follow-up of all events is no longer 
practical. The cosmological results from the Pan-STARRS1 Medium Deep Survey have been published by Rest et al. (2014). The full sample is being published now (Scolnic et al. 2017). The results confirm the cosmological parameters of the previous studies (see Section 2.3).

The Dark Energy Survey (DES) will discover about 4000 SNe Ia. The critical host galaxy redshifts are collected in a separate observing program on the AAT, OzDES (Childress et al. 2017). DES should finish with a superior photometric calibration compared to earlier efforts (SNLS, SDSS) and thus should result in reduced uncertainties. DES will also be able to construct Hubble diagrams and perform cosmological analyses for large subsamples of SNe Ia, equivalent in size and power to SNLS, for the first time, providing a very sensitive test of astrophysical systematic uncertainties.

At lower redshifts, searches for, and studies of, nearby events continue. The $\mathrm{SH}_{0} \mathrm{ES}$ project continues to observe Cepheids in nearby galaxies with well-observed SNe Ia. Gaia will provide a new calibration of the Cepheid period-luminosity calibration by determining exact distances to many Cepheids in the Milky Way. This will further reduce the systematic uncertainty in the determination of $H_{0}$. It appears important to continue to define additional geometric distance indicators to calibrate the SNe Ia.

The Nearby SN Factory has assembled several hundred SNe Ia with detailed observations. The cosmological results are encouraging, although a final publication on $H_{0}$ is still outstanding. A correlation between the peak luminosity and the local star formation rate in the region where the SN occurred has been found (Rigault et al. 2015) and a new method by searching for SN Ia 'twins' at different redshifts has been proposed to improve the relative distance measurements (Fakhouri et al. 2015). The dense sampling has also been used to investigate the local flow field (Feindt et al. 2013).

SNe Ia for the purposes of cosmology have not been the main focus of other nearby searches. The Palomar Transient Factory contributed several important observations of SN Ia and how we can understand the physics of these explosions better. The PESSTO/ePESSTO survey (Smartt et al. 2015) has focused on peculiar objects and has given SNe Ia lower priority. The All-Sky Automated Survey for Supernova (ASAS-SN; Holoien et al. $2017 \mathrm{a}, \mathrm{b}, \mathrm{c}$ ) is searching for very bright, nearby SNe and emphasises the new physics that can be learned from high-cadence observations.

Another promising approach is to observe SNe Ia in the restframe nearinfrared. This has been attempted with $H S T$ through two programs following SNe Ia detected by Pan-STARRS1 and the DES. Because of the decreased systematics even a small fraction of the NIR light curves can yield cosmological parameters that will be competitive. So far, the results of these programs have not been published.

\section{The Future}

Supernova cosmology is well-established. SNe Ia remain the foremost secondary distance indicators for the Hubble constant. There is no other distance indicator in the local universe, which can match the accuracy provided by SNe Ia. It is conceivable that the primary distance indicators might be 
expanded into the Hubble flow but progress has been slow. Megamasers in galaxy cores appeared for a while as the most promising candidate for a precise determination of $H_{0}$, but there have been only very few objects with the required geometry. An alternative are Type II supernovae through the expanding photosphere method and related methods (Nugent and Hamuy 2016 provide an overview of the current developments in this area). Extensions to higher redshifts are starting to appear (Gall et al. 2017, de Jaeger et al. 2017a,b).

Searches for distant SNe Ia will be dominated by near whole-sky, highcadence surveys. The Large Synoptic Survey Telescope (LSST) will find hundreds of thousands of transient candidates every night, and probably in excess of 100,000 SNe Ia after five years. It will provide huge statistical light-curve samples, but spectroscopic follow-up will be limited: a major systematic uncertainty will be the photometric classification (and redshift measurement) of the SN detections. Large multiplex spectrographs, such as $4 \mathrm{MOST}$ at the ESO/VISTA telescope, are the most promising solution and will provide redshifts of host galaxies - very similar to OzDES forDhawan DES - and spectra of thousands of the SNe providing spectroscopic classifications. But even with 4MOST and other facilities, spectroscopic resources will not be available to target all candidate SNe, and photometric classification techniques are therefore critical for LSST SN Ia cosmological analyses. Plans to harness such statistical samples and improve on systematics are being developed. It remains to be demonstrated that these methods will be able to overcome the above challenges.

The space projects Euclid and WFIRST will also be major steps in SN Ia cosmology. Astier et al. (2014) chart an extension program for Euclid (the Dark Energy Supernova Infra-Red Experiment: DESIRE) to build a densely sampled Hubble diagram to $z<1.5$ containing nearly 20000 SNe Ia. Part of this sample would be gathered from the ground, while the high-redshift component would be obtained in a 6-month extension of the Euclid mission with observations dedicated to distant supernovae. SNe Ia are part of the core program of the WFIRST mission. It will provide Hubble diagrams at many different wavelengths and in particular provide the restframe nearinfrared.

The goal must be to measure any time dependence of the equation of state parameter. The uncertainties on a constant $\omega$ in a flat cold dark matter universe have been reduced to very small values. There are many theories, which allow for a variable $\omega$ but constraining any time-dependent $\omega(t)$ has so far been rather inconclusive. The required control on the individual distance measurements are very stringent and have not been achieved so far.

Supernova measurements remain at the core of observational cosmology. They make a unique contribution through their exquisite individual distances. As stellar objects that can be observed across more than half the known universe, they achieve with small samples what needs huge statistics for other objects (weak lensing, redshift surveys, baryonic acoustic oscillations, cosmic microwave background fluctuations) and complement the other methods. 
Acknowledgements B.L. acknowledges support for this work by the Deutsche Forschungsgemeinschaft through the TransRegio Project TRR33 "The Dark Universe".

\section{References}

Amanullah, R., Johansson, J., Goobar, A., et al. 2015, Diversity in extinction laws of Type Ia supernovae measured between 0.2 and $2 \mu \mathrm{m}$, MNRAS, 453, 3300

Astier, P., Balland, C., Brescia, M., et al. 2014, Extending the supernova Hubble diagram to $z \sim 1.5$ with the Euclid space mission, A\&A, 572, A80

Bahcall, N. A., Ostriker, J. P., Perlmutter, S., \& Steinhardt, P. J. 1999, The Cosmic Triangle: Revealing the State of the Universe, Science, 284, 1481

Barone-Nugent, R. L., Lidman, C., Wyithe, J. S. B., et al. 2012, Near-infrared observations of Type Ia supernovae: the best known standard candle for cosmology, MNRAS, 425, 1007

Betoule, M., Kessler, R., Guy, J., et al. 2014, Improved cosmological constraints from a joint analysis of the SDSS-II and SNLS supernova samples, A\&A, 568, A22

Blondin, S., Davis, T. M., Krisciunas, K., et al. 2008, Time Dilation in Type Ia Supernova Spectra at High Redshift, ApJ, 682, 724

Branch, D. 1982, The Hubble diagram for type I supernovae, ApJ, 258, 35

Branch, D., \& Tammann, G. A. 1992, Type Ia supernovae as standard candles, Annual Reviews of Astronomy and Astrophysics, 30, 359

Branch, D. 1998, Type Ia Supernovae and the Hubble Constant, Annual Reviews of Astronomy and Astrophysics, 36, 17

Branch, D., \& Wheeler, J. C. 2017, Supernova Explosions: Astronomy and Astrophysics Library, ISBN 978-3-662-55052-6. Springer-Verlag Germany, 2017

Burns, C. R., Stritzinger, M., Phillips, M. M., et al. 2011, The Carnegie Supernova Project: Light-Curve Fitting with SNooPy, AJ, 141, 19

Childress, M. J., Lidman, C., Davis, T. M., et al. 2017, OzDES multifibre spectroscopy for the Dark Energy Survey: 3-yr results and first data release, MNRAS, 472, 273

Cikota, A., Patat, F., Cikota, S., Spyromilio, J., \& Rau, G. 2017, Common continuum polarization properties: a possible link between proto-planetary nebulae and Type Ia Supernova progenitors, MNRAS, 471, 2111

Conley, A., Sullivan, M., Hsiao, E. Y., et al. 2008, SiFTO: An Empirical Method for Fitting SN Ia Light Curves, ApJ, 681, 482

Conley, A., Guy, J., Sullivan, M., et al. 2011, Supernova Constraints and Systematic Uncertainties from the First Three Years of the Supernova Legacy Survey, ApJS, 192,1

Contardo, G., Leibundgut, B., \& Vacca, W. D. 2000, Epochs of maximum light and bolometric light curves of type Ia supernovae, A\&A, 359, 876

de Jaeger, T., Galbany, L., Filippenko, A. V., et al. 2017, SN 2016jhj at redshift 0.34: extending the Type II supernova Hubble diagram using the standard candle method, MNRAS, 472, 4233

de Jaeger, T., González-Gaitán, S., Hamuy, M., et al. 2017, A Type II Supernova Hubble Diagram from the CSP-I, SDSS-II, and SNLS Surveys, ApJ, 835, 166

Dhawan, S., Leibundgut, B., Spyromilio, J., \& Maguire, K. 2015, Near-infrared light curves of Type Ia supernovae: studying properties of the second maximum, MNRAS, 448, 1345

Dhawan, S., Leibundgut, B., Spyromilio, J., \& Blondin, S. 2016, A reddening-free method to estimate the ${ }^{56} \mathrm{Ni}$ mass of Type Ia supernovae, A\&A, 588, A84

Dhawan, S., Leibundgut, B., Spyromilio, J., \& Blondin, S. 2017, Two classes of fast-declining Type Ia supernovae, A\&A, 602, A118

Dhawan, S., Jha, S. W., \& Leibundgut, B. 2018, Measuring the Hubble constant with Type Ia supernovae as near-infrared standard candles, A\&A, 609, A72

Dilday, B., Howell, D. A., Cenko, B., et al. (2012), PTF 11kx: A Type Ia Supernova with a Symbiotic Nova Progenitor, Science, 237, 942 
Fakhouri, H. K., Boone, K., Aldering, G., et al. 2015, Improving Cosmological Distance Measurements using Twin Type Ia Supernovae, ApJ, 815, 58

Feindt, U., Kerschhaggl, M., Kowalski, M., et al. 2013, Measuring cosmic bulk flows with Type Ia supernovae from the Nearby Supernova Factory, A\&A, 560, A90

Filippenko, A. V., Richmond, M. W., Matheson, T., et al. 1992, The peculiar Type Ia SN 1991T - Detonation of a white dwarf?, ApJL, 384, L15

Fink, M., Kromer, M., Seitenzahl, I. R., et al. 2014, Three-dimensional pure deflagration models with nucleosynthesis and synthetic observables for Type Ia supernovae, MNRAS, 438, 1762

Foley, R. J., Challis, P. J., Chornock, R., et al. 2013, Type Iax Supernovae: A New Class of Stellar Explosions, ApJ, 767, 57

Freedman, W. L., Madore, B. F., Gibson, B. K., et al. 2001, Final Results from the Hubble Space Telescope Key Project to Measure the Hubble Constant, ApJ, 553,47

Freedman, W. L., Burns, C. R., Phillips, M. M., et al. 2009, The Carnegie Supernova Project: First Near-Infrared Hubble Diagram to $z \sim 0.7$, ApJ, 704, 1036

Gall, E. E. E., Kotak, R., Leibundgut, B., et al. 2017, An updated Type II supernova Hubble diagram, arXiv:1705.10806

Garnavich, P., 2017, Discovery of Cosmic Acceleration, in Handbook of Supernovae, A.W. Alsabti, P. Murdin (eds.), Springer, DOI:10.1007/978-3-319-20794-0_1041

Goldhaber, G., Groom, D. E., Kim, A., et al. 2001, Timescale Stretch Parameterization of Type Ia Supernova B-Band Light Curves, ApJ, 558, 359

Goobar, A., \& Perlmutter, S. 1995, Feasibility of Measuring the Cosmological Constant Lambda and Mass Density Omega Using Type Ia Supernovae, ApJ, 450, 14

Goobar, A., \& Leibundgut, B. 2011, Supernova Cosmology: Legacy and Future, Annual Review of Nuclear and Particle Science, 61, 251

Guy, J., Astier, P., Baumont, S., et al. 2007, SALT2: using distant supernovae to improve the use of type Ia supernovae as distance indicators, A\&A, 466, 11

Hamuy, M., Phillips, M. M., Suntzeff, N. B., et al. 1996, The Hubble Diagram of the Calán/Tololo Type Ia Supernovae and the Value of $H_{0}$, AJ, 112, 2398

Holoien, T. W.-S., Stanek, K. Z., Kochanek, C. S., et al. 2017, The ASAS-SN bright supernova catalogue - I. 2013-2014, MNRAS, 464, 2672

Holoien, T. W.-S., Brown, J. S., Stanek, K. Z., et al. 2017, The ASAS-SN bright supernova catalogue - II. 2015, MNRAS, 467, 1098

Holoien, T. W.-S., Brown, J. S., Stanek, K. Z., et al. 2017, The ASAS-SN bright supernova catalogue - III. 2016, MNRAS, 471, 4966

Howell, D. A., Sullivan, M., Nugent, P. E., et al. 2006, The type Ia supernova SNLS03D3bb from a super-Chandrasekhar-mass white dwarf star, Nature, 443, 308

Humphreys, E. M. L., Reid, M. J., Moran, J. M., Greenhill, L. J., \& Argon, A. L. 2013, Toward a New Geometric Distance to the Active Galaxy NGC 4258. III. Final Results and the Hubble Constant, ApJ, 775, 13

Jha, S., Riess, A. G. \& Kirshner, R. P. 2007, Improved Distances to Type Ia Supernovae with Multicolor Light-Curve Shapes: MLCS2k2, ApJ, 659, 122

Jha, S. W., 2017, Type Iax Supernovae, in Handbook of Supernovae, A.W. Alsabti, P. Murdin (eds.), Springer, DOI: 10.1007/978-3-319-20794-0_42-1

Jones, D. O., Scolnic, D. M., Riess, A. G., et al. 2018, Measuring Dark Energy Properties with Photometrically Classified Pan-STARRS Supernovae. II. Cosmological Parameters, arXiv:1710.00846

Kerzendorf, W. E., Strampelli, G., Shen, K. J., et al. 2017, A Search for a Surviving White Dwarf Companion in SN 1006, arXiv:1709.06566

Krisciunas, K., Phillips, M. M., \& Suntzeff, N. B. 2004, Hubble Diagrams of Type Ia Supernovae in the Near-Infrared, ApJL, 602, L81

Krisciunas, K., 2016, The Infrared Hubble Diagram of Type Ia Supernovae, in Handbook of Supernovae, A.W. Alsabti, P. Murdin (eds.), Springer, DOI: 10.1007/978-3-319-20794-0_103-1

Leibundgut, B. 2001, Cosmological Implications from observations of Type Ia Supernovae, Annual Reviews of Astronomy and Astrophysics, 39, 67 
Leibundgut, B. 2008, Supernovae and Cosmology, General Relativity and Gravitation, 40, 221

Leibundgut, B., 2016, History of Supernovae as Distance Indicators, in Handbook of Supernovae, A.W. Alsabti, P. Murdin (eds.), Springer, DOI:10.1007/978-3319-20794-0_99-1

Leibundgut, B., Schommer, R., Phillips, M., et al. 1996, Time Dilation in the Light Curve of the Distant Type Ia Supernova SN 1995K, ApJL, 466, L21

Li, W., Filippenko, A. V., Chornock, R., et al. 2003, SN 2002cx: The Most Peculiar Known Type Ia Supernova, PASP, 115, 453

Li, W., Filippenko, A. V., Gates, E., et al. 2001, The Unique Type Ia Supernova 2000cx in NGC 524, PASP, 113, 1178

Li, W., Bloom, J. S., Podsiadlowski, P., et al. 2011, Exclusion of a luminous red giant as a companion star to the progenitor of supernova SN 2011fe, Nature, 480,348

Maguire, K., Sullivan, M., Patat, F., et al. 2013, A statistical analysis of circumstellar material in Type Ia supernovae, MNRAS, 436, 222

Maguire, K., 2016, Type Ia Supernovae, in Handbook of Supernovae, A.W. Alsabti, P. Murdin (eds.), Springer, DOI:10.1007/978-3-319-20794-0_36-1

Mandel, K. S., Scolnic, D. M., Shariff, H., Foley, R. J., \& Kirshner, R. P. 2017, Star Formation, Supernovae, Iron, and : Consistent Cosmic and Galactic Histories, ApJ, 842, 93

Narayan, G., Rest, A., Tucker, B. E., et al. 2016, Light Curves of 213 Type Ia Supernovae from the ESSENCE Survey, ApJS, 224, 3

Nugent, P., Phillips, M., Baron, E., Branch, D., \& Hauschildt, P. 1995, Evidence for a Spectroscopic Sequence among Type 1a Supernovae, ApJL 455, 147

Nugent, P., Hamuy, M. 2016, Cosmology with Type IIP Supernovae, in Handbook of Supernovae, A.W. Alsabti, P. Murdin (eds.), Springer, DOI:10.1007/978-3319-20794-0_108-1

Nugent, P. E., Sullivan, M., Cenko, S. B., et al. 2011, Supernova SN 2011fe from an exploding carbon-oxygen white dwarf star, Nature, 480, 344

Patat, F., Chandra, P., Chevalier, R., et al. 2007, Detection of Circumstellar Material in a Normal Type Ia Supernova, Science, 317, 924

Perlmutter, S., Aldering, G., Goldhaber, G., et al. 1999, Measurements of and from 42 High-Redshift Supernovae, ApJ, 517, 565

Perlmutter, S., \& Schmidt, B. P. 2003, Measuring Cosmology with Supernovae, in Supernovae and Gamma-Ray Bursters, Lecture Notes in Physics 598, 195

Phillips, M. M. 1993, The absolute magnitudes of Type Ia supernovae, ApJL, 413, L105

Phillips, M. M., Wells, L. A., Suntzeff, N. B., et al. 1992, SN 1991T - Further evidence of the heterogeneous nature of type Ia supernovae, AJ, 103, 1632

Phillips, M. M., Li, W., Frieman, J. A., et al. 2007, The Peculiar SN 2005hk: Do Some Type Ia Supernovae Explode as Deflagrations?, PASP, 119, 360

Phillips, M. M., Simon, J. D., Morrell, N., et al. 2013, On the Source of the Dust Extinction in Type Ia Supernovae and the Discovery of Anomalously Strong Na I Absorption, ApJ 779, 38

Phillips, M.M., Burns, C.R., 2016, The Peak LuminosityDecline Rate Relationship for Type Ia Supernovae, in Handbook of Supernovae, A.W. Alsabti, P. Murdin (eds.), Springer, DOI: 10.1007/978-3-319-20794-0_100-1

Planck Collaboration, Ade, P. A. R., Aghanim, N., et al. 2016, Planck 2015 results. XIII. Cosmological parameters, A\&A, 594, A13

Rest, A., Scolnic, D., Foley, R. J., et al. 2014, Cosmological Constraints from Measurements of Type Ia Supernovae Discovered during the First $1.5 \mathrm{yr}$ of the Pan-STARRS1 Survey, ApJ, 795, 44

Riess, A. G., Filippenko, A. V., Leonard, D. C., et al. 1997, Time Dilation from Spectral Feature Age Measurements of Type Ia Supernovae, AJ, 114, 722

Riess, A. G., Filippenko, A. V., Challis, P., et al. 1998, Observational Evidence from Supernovae for an Accelerating Universe and a Cosmological Constant, AJ, 116, 1009 
Riess, A. G., Macri, L. M., Hoffmann, S. L., et al. 2016, A 2.4\% Determination of the Local Value of the Hubble Constant, ApJ, 826, 56

Riess, A. G., 2016, Confirming Cosmic Acceleration in the Decade that Followed from SNe Ia at $z>1$, in Handbook of Supernovae, A.W. Alsabti, P. Murdin (eds.), Springer, DOI:10.1007/978-3-319-20794-0_105-1

Rigault, M., Aldering, G., Kowalski, M., et al. 2015, Confirmation of a Star Formation Bias in Type Ia Supernova Distances and its Effect on the Measurement of the Hubble Constant, ApJ, 802, 20

Saha, A., Macri, L.M., 2016, The Hubble Constant from Supernovae, in Handbook of Supernovae, A.W. Alsabti, P. Murdin (eds.), Springer, DOI:10.1007/978-3319-20794-0_102-1

Sandage, A. 1961, The Ability of the 200-inch Telescope to Discriminate Between Selected World Models, ApJ, 133, 355

Sandage, A. R. 1970, Cosmology: a search for two numbers, Physics Today, 23, 34

Scalzo, R. A., Ruiter, A. J., \& Sim, S. A. 2014, The ejected mass distribution of Type Ia supernovae: a significant rate of non-Chandrasekhar-mass progenitors, MNRAS, 445, 2535

Scalzo, R., Aldering, G., Antilogus, P., et al. 2014, Type Ia supernova bolometric light curves and ejected mass estimates from the Nearby Supernova Factory, MNRAS, 440, 1498

Schmidt, B. P., Suntzeff, N. B., Phillips, M. M., et al. 1998, The High-Z Supernova Search: Measuring Cosmic Deceleration and Global Curvature of the Universe Using Type Ia Supernovae, ApJ, 507, 46

Scolnic, D., Rest, A., Riess, A., et al. 2014, Systematic Uncertainties Associated with the Cosmological Analysis of the First Pan-STARRS1 Type Ia Supernova Sample, ApJ 795, 45

Scolnic, D. M., \& Kessler, R. 2016, Measuring Type Ia Supernova Populations of Stretch and Color and Predicting Distance Biases, ApJ 822, 35L

Scolnic, D. M., Jones, D. O., Rest, A. et al. 2017, The Complete Lightcurve Sample of Spectroscopically Confirmed Type Ia Supernovae from PanSTARRS1 and Cosmological Constraints from The Combined Pantheon Sample, arXiv: 1710.00845

Shariff, H., Jiao, X., Trotta, R., \& van Dyk, D. A. 2016, BAHAMAS: New Analysis of Type Ia Supernovae Reveals Inconsistencies with Standard Cosmology, ApJ, 827,1

Smartt, S. J., Valenti, S., Fraser, M., et al. 2015, PESSTO: survey description and products from the first data release by the Public ESO Spectroscopic Survey of Transient Objects, A\&A, 579, A40

Stanishev, V., Goobar, A., Amanullah, R., et al. 2015, Type Ia Supernova Cosmology in the Near-Infrared, arXiv:1505.07707

Sternberg, A., Gal-Yam, A., Simon, J. D., et al. 2011, Circumstellar Material in Type Ia Supernovae via Sodium Absorption Features, Science 333, 856

Sternberg, A., Gal-Yam, A., Simon, J. D., et al. 2014, Multi-epoch high-spectralresolution observations of neutral sodium in 14 Type Ia supernova, MNRAS, 443,1849

Stritzinger, M., \& Leibundgut, B. 2005, Lower limits on the Hubble constant from models of type Ia supernovae, A\&A, 431, 423

Stritzinger, M., Leibundgut, B., Walch, S., \& Contardo, G. 2006, Constraints on the progenitor systems of type Ia supernovae, A\&A, 450, 241

Taubenberger, S., 2016, The Extremes of Thermonuclear Supernovae, in Handbook of Supernovae, A.W. Alsabti, P. Murdin (eds.), Springer, DOI:10.1007/978-3319-20794-0_31-1

Taubenberger, S., Benetti, S., Childress, M., et al. 2011, High luminosity, slow ejecta and persistent carbon lines: SN $2009 \mathrm{dc}$ challenges thermonuclear explosion scenarios, MNRAS, 412, 2735

Tripp, R. 1998, A two-parameter luminosity correction for Type Ia supernovae, A\&A, 331, 815

Wilson, O. C. 1939, Possible Applications of Supernovae to the Study of the Nebular Red Shifts, ApJ, 90, 634 